\title{
The Influence of the Gait-Related Arm Swing on Elevation Gain Measured by Sport Watches
}

\author{
by \\ Rahel Ammann ${ }^{1,2}$, Wolfgang Taube ${ }^{2}$, Matthias Neuhaus ${ }^{1}$, Thomas Wyss ${ }^{1}$
}

The elevation gain is an important contributor to the total workload in endurance sports. The purpose of this study was to evaluate the influence of the arm swing on elevation gain in three sport watches (Garmin ${ }^{\circledR}$ Forerunner 910XT, Polar ${ }^{\circledR}$ RS800CX and Suunto ${ }^{\circledR}$ Ambit2) on a flat $400 \mathrm{~m}$ outdoor track. Altogether, a total of 120 repetitions of 1,200 $\mathrm{m}$ were performed at self-selected speeds corresponding to strolling, walking, jogging and running. During the assessment two devices of each sport watch, one secured on the hip and one on the wrist, were worn by the participants. A small but significant (effect size $=.39 ; p<.001$ ) influence of the arm swing on elevation was revealed in all sport watches. Elevation indication errors recorded on the wrist were significantly larger than the ones recorded on the hip (4.0-7.4 vs. 1.2-5.7 $m$ per 1,200 m; $<$.05). Furthermore, when wearing the devices on the wrist, errors in elevation indication increased when gait speed increased. Users should be aware that wearing the devices on the hip can significantly decrease measurement errors. This might be especially relevant for activities with high dynamics, such as jogging and running.

Key words: workload, change in altitude, running.

\section{Introduction}

Most elite athletes in endurance sports are using commercial sport watches to monitor their physiological workload (Fleming et al., 2010). To objectively quantify the workload, different all-inone devices may be found on the market; these devices capture exercise duration, speed, distance, heart rate and energy expenditure. In addition, some devices provide information on altitude, which is an important measure for many outdoor activities. For example, orienteers, mountain runners, mountain bikers, cross-country skiers or simply active people may wish to have feedback about the elevation gain in order to monitor, quantify and evaluate the workload (Fleming et al., 2010). In order to quantify the impact of elevation gain on the workload, athletes can translate vertical distance (change in elevation) into horizontal distance. Recently, a ratio of 1 to 8 in men and 1 to 10 in women was proposed, meaning that $125 \mathrm{~m}$ and $100 \mathrm{~m}$ of vertical distance, respectively, equal the exercise duration of 1,000 $\mathrm{m}$ of horizontal distance (Scarf, 2007).

To assess elevation gain during sports, two main measurement systems exist. Firstly, devices with a barometric altimeter compute the atmospheric pressure into an amount of altitude and compare it with a reference altitude (Immanuvel Arokia James and Sudheer, 2011). These systems are based on decreases in atmospheric pressure that correspond to an increasing sea level. Hence, at an elevated altitude, air pressure reduces; at low altitudes, air pressure raises. Secondly, there are also devices with an integrated global positioning system (GPS), a technology that relies on access to the network of 24 satellites. These satellites circle the earth in a very precise orbit and transmit signals that the device with the GPS can receive (Terrier

1 - Swiss Federal Institute of Sport Magglingen SFISM, Magglingen, Switzerland.

2- University of Fribourg, Department of Medicine, Movement and Sport Science, Fribourg, Switzerland. 
and Schutz, 2005). Based on the time a signal was transmitted by a satellite with the time it was received by the GPS device, the user's location, such as the altitude, can be determined. Both measurement systems can be affected by weather (e.g., temperature) and/or environmental (e.g., buildings) conditions (Immanuvel Arokia James and Sudheer, 2011; Terrier and Schutz, 2005). However, knowledge about the weaknesses of the measurement system does not provide insight into the amount of error. As long as no scientific evaluation has been conducted, the data these measurement systems provide should be interpreted with considerable caution (Düking et al., 2016).

Market leaders for all-in-one devices in sports are manufacturers such as Garmin ${ }^{\circledR}$ (Garmin Ltd., Southampton, UK), Polar ${ }^{\circledR}$ (Polar Electro Oy, Kempele, Finland) and Suunto ${ }^{\circledR}$ (Suunto, Valimotie, Finland) (Fleming et al., 2010). In a study conducted with GPS based devices installed on the roof top of a car and on cyclists' handlebars, the measurement of elevation gain was relatively consistent within the same device types (Menaspa et al., 2014). However, they did not assess concurrent validity. Moreover, some of the above mentioned manufacturers' products are commercially available as sport watches; thus, they are designed to be worn on the wrist. Yet, while wearing the watch on the wrist is very userfriendly, it may have some inherent constraints with regard to elevation indication compared to steady placements, e.g., on handlebars. During bipedal locomotion, such as walking and running, an automatic arm swing sets in as it stabilises the posture of the body (Anderson, 1996; Williams and Cavanagh, 1987). Both the frequency and the amplitude of the arm swing depend on gait speed (Thielemans et al., 2014). Thus, slow gait speeds result in low frequencies and small amplitudes, which increase with running and sprinting. The frequency and the amplitude are also both dependent on body height, as taller people typically have longer legs and arms, which they move with lower frequencies but larger amplitudes when travelling at the same speed as shorter people.

The aim of this study was to investigate the influence of the gait-related arm swing on elevation gain assessed by sport watches. For this purpose, elevation measures that were simultaneously obtained on the hip and the wrist were compared.

\section{Material and Methods}

\section{Participants}

Three men with body height of $1.68,1.79$ and $1.87 \mathrm{~m}$ (age: $25.5 \pm 1.3$ years; body mass: $77.3 \pm 4.6 \mathrm{~kg})$ and all without any walking disabilities, were recruited to participate in this study. The aim of the study could have been investigated with one participant only, performing many repetitions. However, we wanted to further pay attention to the participants' heights and thus, we had recruited three participants considerably different in body height and let them repeat the trials 40 times each. All subjects were recreational runners, practicing endurance sports more than 300 minutes per week. Moreover, they were former middle- and long-distance runners with a history of athletic training. Informed consent was obtained from all participants after familiarization with the procedure. The study protocol was approved by the Institutional Review Board of the Swiss Federal Office of Sport, in the spirit of the Declaration of Helsinki.

\section{Measures}

The devices used in this study were the Garmin $^{\circledR}$ Forerunner XT910 (GF), the Polar $^{\circledR}$ RS800CX (PRS) and the Suunto ${ }^{\circledR}$ Ambit2 (SA). According to the information on the respective websites, the GF calculates elevation using a barometric altimeter but applies GPS data in case the barometric altimeter starts to report false values due to environmental conditions, such as weather events or pressurised areas (Garmin Ltd.). The PRS has a built-in barometer (Polar Electro Oy), whereas the SA provides elevation indication based on barometric information continuously corrected by the GPS (Suunto). However, we cannot make any statements about the underlying mechanisms of how elevation gain is derived exactly, as none of the manufacturer provided such information upon enquiry. Furthermore, a tracking device (Suunto ${ }^{\circledR}$ GPS Track POD) to measure mean speed and an accelerometer (GT3X, ActiGraph ${ }^{\circledast}$, Pensacola, Florida) to calculate step frequency, which was equated to the frequency of the arm swing, were included in this study. To control for weather influences, data from an air pressure gauge (GDH 
200-14, GHM Messtechnik, Regenstauf, Germany) were recorded.

\section{Procedures}

The participants were asked to complete trials of $1,200 \mathrm{~m}$ on a flat $400 \mathrm{~m}$ synthetic track, each time at a self-selected speed corresponding to strolling, walking, jogging and running. Standardised instructions were given for the selfselected speeds to ensure natural movement patterns: for strolling, "walk gently, as you would enjoy a sunny day alongside the lake"; for walking, "brisk walking, as you would be rushing to catch the bus, though without running"; for jogging, "easy jogging, that you could talk to a companion or jog for $60 \mathrm{~min}$ "; and for running, "try to complete the 1,200 $\mathrm{m}$ as fast as possible, though at a steady speed". Each participant was tested on ten occasions to account for different weather conditions and times of the day (Menaspa et al., 2014). Prior to each trial, the devices were calibrated as recommended by the manufacturers to the baseline elevation of $857 \mathrm{~m}$ above the sea level. The epoch duration was set to $1 \mathrm{~s}$. The participants performed the 1,200 m trial four times each test day, once per gait speed, meaning ten visits per participant, four trials per visit, resulting in a total of 40 trials per participant. In the course of every measurement, six devices, two from each manufacturer, were worn simultaneously. One device of each manufacturer was worn on the wrist, and the other one was worn on the hip, tightly fixed to a belt (Figure 1). The order of the sport watches around the hip and location on wrist, e.g., two devices on the left and one device on the right, was randomised. The GPS track pod was strapped to the upper arm and the accelerometer was placed on the hip. Participants were instructed to stay still behind the starting line of the $400 \mathrm{~m}$ track. Then the examiner started the devices before the participant began each 1,200 m trial. After completion, participants stopped exactly behind the finish line of the $400 \mathrm{~m}$ track and stood still until the examiner stopped all devices. At least two minutes of recovery were allowed between trials. At the start and end of each trial, air pressure values were noted to evaluate fluctuations in weather conditions between and within-trials. Data from the devices were uploaded after every test day to the corresponding webpage or software (Garmin ${ }^{\circledR}$
Connect, Polar ${ }^{\circledR}$ ProTrainer 5 and Movescount, respectively) and manually post-processed.

\section{Statistical analysis}

The statistical analyses were executed with Microsoft Excel (2011) and SPSS 22.0 (IBM Corporation, Armonk, NY, USA). Data from each device were analysed manually in 1-s epochs. As the track was flat, every recorded elevation gain was added up to a total elevation indication error (EIE) per $1,200 \mathrm{~m}$. Normality of the data was rejected because the ratios of the skewness to the standard error of skewness exceeded \pm 2.0 (Field, 2012). Subsequently, differences in EIEs between the three manufacturers were analysed by means of Friedman's analysis of variance. To analyse whether the gait-related arm swing influenced elevation indication, differences in EIEs between positions (hip vs. wrist) of the same device were assessed by Wilcoxon signed ranks tests. The effect sizes (ES) were calculated based on the $z$-scores (Cohen, 1988; Field, 2012). The ES of $<0.2$, between 0.2-0.5, between 0.5-0.8, and $>0.8$ were considered trivial, small, moderate and large, respectively. Linear regression analysis was executed to evaluate if gait speed, body height and weather conditions between and within-trials were predictors of the influence of the arm swing on EIE. The mean difference in EIE between the hip and the wrist position was regarded as the influence of the gait-related arm swing, and therefore, entered as a dependent variable. Prior to that, the assumption of linearity and homoscedasticity was accepted (Field, 2012). Data were illustrated by means of boxplots and any data point more than 1.5 times the interquartile range below the first or above the third quartile, respectively, was defined as an outlier (Field, 2012). Inter-trial speed was investigated by coefficients of variation (CV) for strolling, walking, jogging and running, respectively. The results were considered significant if $p \leq .05$.

\section{Results}

A total of 120 datasets were analysed. All devices showed EIEs over the course of 1,200 m accomplished on the flat outdoor track $(2.6 \pm 4.3 \mathrm{~m}, 3.9 \pm 2.7 \mathrm{~m}$ and $6.5 \pm 3.1 \mathrm{~m}$ in the GF, the PRS and the SA, respectively). Significant differences between devices were found $\left(\chi^{2}(2)=187.16, \quad p<.001\right): \quad$ the $\quad$ SA recorded significantly larger absolute EIEs compared to the 
GF and the PRS, and the PRS showed significantly larger absolute EIEs than the GF. Devices worn on the wrist recorded significantly larger EIEs in all three device types (all $p$-values <.05) compared to the data assessed on the hip (Table 1).

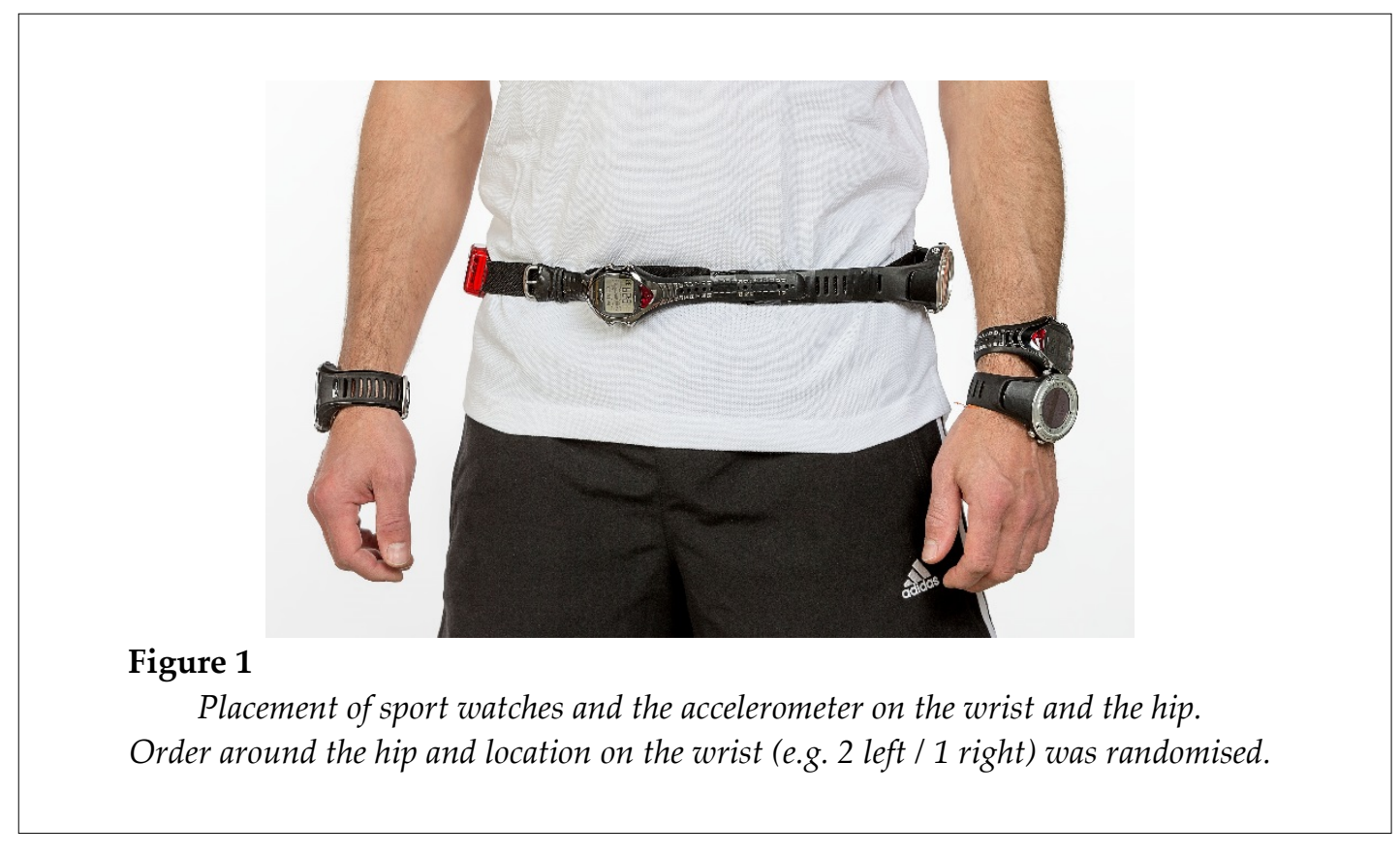

Table 1

Total elevation indication errors per 1,200 $m$ as means \pm standard deviations assessed on a flat $400 \mathrm{~m}$ outdoor track. Overall data $(n=120)$ are subdivided

\begin{tabular}{|c|c|c|c|c|}
\hline $\begin{array}{l}\text { Speed } \\
\left(\mathrm{m} \cdot \mathrm{s}^{-1}\right) \\
(\text { steps per second) }\end{array}$ & Position & $\begin{array}{l}\text { Garmin }{ }^{\circledR} \\
\text { Forerunner } \\
\text { 910XT } \\
(\mathrm{m}) \\
\end{array}$ & $\begin{array}{l}\text { Polar }^{\circledR} \\
\text { RS800CX } \\
\text { (m) }\end{array}$ & $\begin{array}{l}\text { Suunto }^{\circledast} \\
\text { Ambit2 } \\
\text { (m) }\end{array}$ \\
\hline Overall & hip & $1.2 \pm 2.2$ & $3.4 \pm 2.3$ & $5.7 \pm 2.7$ \\
\hline $2.88 \pm .99$ & wrist & $4.0 \pm 5.4$ & $4.5 \pm 3.0$ & $7.4 \pm 3.2$ \\
\hline \multirow[t]{2}{*}{$2.35 \pm .42$} & $p$ & .000 & .003 & .000 \\
\hline & ES & .51 & .27 & .39 \\
\hline Strolling & hip & $1.3 \pm 1.7$ & $3.9 \pm 2.3$ & $6.9 \pm 3.0$ \\
\hline $1.77 \pm .08$ & wrist & $1.6 \pm 3.6$ & $4.7 \pm 2.3$ & $8.1 \pm 2.8$ \\
\hline \multirow[t]{2}{*}{$2.02 \pm .24$} & $p$ & .774 & .180 & .141 \\
\hline & ES & .05 & .25 & .27 \\
\hline Walking & hip & $0.7 \pm 1.4$ & $3.1 \pm 2.4$ & $6.6 \pm 2.7$ \\
\hline $2.26 \pm .15$ & wrist & $3.4 \pm 5.4$ & $3.7 \pm 2.6$ & $8.5 \pm 3.5$ \\
\hline \multirow[t]{2}{*}{$2.06 \pm .32$} & $p$ & .003 & .175 & .039 \\
\hline & ES & .54 & .25 & .38 \\
\hline Jogging & hip & $0.9 \pm 1.8$ & $3.6 \pm 1.9$ & $4.5 \pm 1.8$ \\
\hline $3.27 \pm .28$ & wrist & $4.8 \pm 5.7$ & $3.8 \pm 2.6$ & $6.2 \pm 3.0$ \\
\hline \multirow[t]{2}{*}{$2.59 \pm .24$} & $p$ & .000 & .841 & .015 \\
\hline & ES & .67 & .04 & .45 \\
\hline Running & hip & $1.9 \pm 3.3$ & $3.2 \pm 2.5$ & $4.9 \pm 2.5$ \\
\hline $4.22 \pm .46$ & wrist & $6.2 \pm 5.6$ & $5.6 \pm 3.8$ & $6.6 \pm 3.0$ \\
\hline \multirow[t]{2}{*}{$2.73 \pm .31$} & $p$ & .001 & .006 & .002 \\
\hline & ES & .63 & .51 & .57 \\
\hline
\end{tabular}

p-values and ES represent comparisons between the hip and the wrist; $E S=$ effect size $($ Cohen's d). 


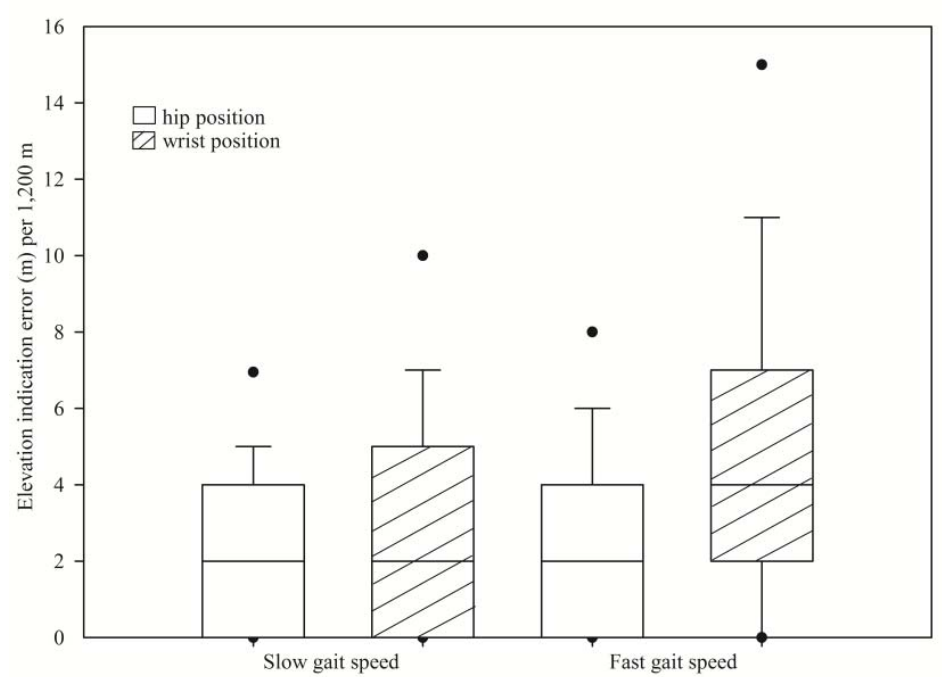

Figure 2

Data presentation in boxplots separated for slow (strolling and walking) and fast (jogging and running) gait speed according to position on body.

For each boxplot the middle line represents the median value, the lower and upper limits represent the interquartile range, the error bars indicate the range and the dots denote the $5^{\text {th }}$ and $95^{\text {th }}$ percentiles.

Table 2

Gait speed and step frequency of overall data $(n=120)$ according to participants' body heights.

\begin{tabular}{|c|c|c|c|c|}
\hline & \multicolumn{3}{|c|}{ Body height } & \multirow[b]{2}{*}{$F^{a}$} \\
\hline & $1.68 \mathrm{~m}$ & $1.79 \mathrm{~m}$ & $1.87 \mathrm{~m}$ & \\
\hline $\begin{array}{l}\text { Speed } \\
\left(\mathrm{m} \cdot \mathrm{s}^{-1}\right)\end{array}$ & $2.94 \pm 1.01$ & $2.75 \pm 0.96$ & $2.94 \pm 1.00$ & .463 \\
\hline $\begin{array}{l}\text { Step frequency } \\
\text { (steps per second) }\end{array}$ & $2.34 \pm 0.41$ & $2.30 \pm 0.51$ & $2.40 \pm 0.31$ & .528 \\
\hline
\end{tabular}

The ESs of the difference in EIEs between the hip and the wrist were small in the PRS and the SA (.27 and .39, respectively) and moderate in the GF (.51).

The linear regression analysis showed that faster gait speed and shorter body height were significantly related to larger mean differences in EIEs between the hip and the wrist $(t(356)=3.67$, $p<.001$ and $t(356)=-1.98, p=.049$, respectively; Figure 2). The weather conditions between and within-trials did not influence EIE. Faster gait speed was significantly $(F(3,119)=49.124, p<.001)$ associated with increased step frequency. However, self-selected gait speeds and corresponding mean step frequencies did not significantly differ among the three participants 
varying in body heights (Table 2). The relative amount of outliers reported for the device position on the hip or wrist were $6.7 / 15.0 \%$, $5.8 / 3.3 \%$ and $6.7 / 5.8 \%$ for the GF, the PRS and the SA, respectively. All subjects accomplished the self-paced courses at very steady inter-trial speeds with CVs of $5.7,6.0,4.1$ and $3.8 \%$ for strolling, walking, jogging and running, respectively.

\section{Discussion}

The present study evaluated differences in elevation gain measures between sport watches worn either on the hip or on the wrist. All tested devices, the GF, the PRS and the SA, recorded significantly larger EIEs when worn on the wrist compared to when the same device was worn on the hip. As recordings from the hip and the wrist were taken simultaneously with two identical devices, it is not possible that differences in task execution could explain this phenomenon. It can rather be concluded that the gait-related arm swing negatively affected the accuracy of elevation indication. More specifically, the arm swing led to overestimation of the actual elevation gain. This overestimation was further enlarged by increasing gait speed, which is well depicted in Figure 2. The reason for this enlarged overestimation might be related to an enhancement in arm swing frequency and/or an enhancement in arm swing amplitude with faster gait speeds (Anderson, 1996; Thielemans et al., 2014; Williams and Cavanagh, 1987). These variables are known to vary with changes in gait speed. We recorded only step frequency, which should also precisely reflect arm swing frequency, and we could indeed show an increase in step frequency with faster speeds. Unfortunately, no quantifiable data about the arm swing amplitude are available, as it was not assessed. We can only speculate that both factors, i.e., arm swing frequency and amplitude, contributed to the enlarged EIEs with increased gait speed when the data were recorded on the wrist.

However, not only gait speed but also the body height of the participants influenced the EIE. Again, it might be argued that body height influences both arm swing frequency and amplitude. We assumed that shorter participants should reveal higher arm swing frequencies but smaller amplitudes at a certain speed than taller people. Surprisingly, this was not the case, as step frequency and self-selected gait speeds did not significantly differ between the three participants nor could a trend be detected to reinforce this assumption. Based on this lack of conjunction between body height and step frequency, and the fact that not only the EIE on the wrist, but also the one recorded on the hip increased in shorter participants, it seems reasonable to assume that the individual gait style might have affected EIE. In this study, the shorter participants probably rebounded more from the ground to achieve step lengths equal to those of the tallest participant, leading to greater vertical displacements of the centre of mass and thus, even greater overestimation of the elevation gain (Anderson, 1996). However, whether this assumption holds on or to draw final conclusions on the influence of body height, more research is recommended.

The interesting question now is to what extent the EIEs obtained during this study are functionally relevant. For example, an extrapolation of the largest mean EIE per any gait speed and per manufacturer over the distance of $10,000 \mathrm{~m}$ would result in absolute EIEs ranging from $15.8-57.5 \mathrm{~m}$ when measured on the hip and $46.7-70.8 \mathrm{~m}$ when measured on the wrist. Previously, a typical cut-off point between good and moderate accuracy was made at $5 \%$ of measurement error (Coutts and Duffield, 2010; Duthie et al., 2003; Roberts et al., 2006). Taking the literature of Scarf (2007) into account and the assertion that $125 \mathrm{~m}$ (men) and $100 \mathrm{~m}$ (women) of climb, respectively, equal the exercise duration of $1,000 \mathrm{~m}$ of horizontal travel, the impact of EIEs on the total workload for men would be $<5 \%$ in the GF and the PRS in both positions and in the SA only when worn on the hip (hip / wrist: $1.3 / 4.1 \%$, $2.6 / 3.7 \%, 4.6 / 5.7 \%$ in the GF, the PRS and the $\mathrm{SA}$, respectively). For women, climb has a larger effect on exercise duration; only the use of the PRS would result in an impact of EIEs on total exercise duration of $<5 \%$ in both positions (hip / wrist: $3.3 / 4.7 \%)$. For the GF, the error impact would be $>5 \%$ when the device is worn on the wrist $(5.2 \%)$. Furthermore, when using the SA, total exercise duration would be overestimated by $>5 \%$ for hip and wrist placements (5.8 / 7.1\%). Moreover, it has been previously demonstrated that some runners increase the arm swing during exhausting 45 minute runs, indicating poorer running economy 
with fatigue (Strohrmann et al., 2012). Although we could not integrate this aspect in the current study, as each trial lasted only a few minutes, it must nevertheless be considered that EIEs might be enlarged even more with increasing fatigue when devices are worn on the wrist. Hence, the overestimation of exercise duration, and therefore the total workload, might be even larger than in the prior described extrapolation.

In general, compared to the GF and the PRS, the SA showed the largest absolute EIEs when worn in either position. However, the GF displayed largest differences in EIEs between positions, meaning that wearing the GF on the wrist resulted in much larger errors than wearing the same device on the hip. This finding is underlined by the $15.0 \%$ outliers registered by the GF when worn on the wrist, which was considerable more than, e.g., by the PRS with $3.3 \%$. There was no obvious reason due to external influences that could explain these outliers. Moreover, it was never the case that outliers were reported in all three sport watches during the same trial. For this reason and the fact that the end-user would like to have accurate data in every single training session or competition, all results from all trials and all manufacturers were included in the analyses. The PRS was the least sensitive to the arm swing compared to the other sport watches, with the smallest ESs. At the same time, the PRS was the only device that calculated elevation indication solely by means of barometric measures, whereas the measures from the GF and the SA were based on barometric information and on the GPS. It may therefore be assumed that the
GPS negatively influenced the elevation assessments. Although the outdoor track was free of any restrictive surroundings such as trees, buildings or walls that could have interfered with GPS signals.

A limitation of the present study was the small sample size. With only three participants, though considerably different in body height (1.68, 1.79 and $1.87 \mathrm{~m}$, respectively), conclusions drawn about the influence of body heights on EIEs must be treated with caution. However, the main aim of the study could have been investigated with one participant only. Further studies are required to investigate the accuracy of elevation indication assessed by sport watches in environments with authentic ascents and descents, and with larger samples that include diverging body heights.

\section{Conclusion}

This study demonstrated that the accuracy of elevation indication at different gait speeds assessed by three sport watches was moderate to good with mean EIEs of $3.5 \pm 3.0 \mathrm{~m}$ and $5.3 \pm 4.2 \mathrm{~m}$ per 1,200 $\mathrm{m}$ when worn on the hip and the wrist, respectively. As a consequence of the present study's results, it appears that the basic error in elevation indication reported by the tested sport watches was reinforced by the common placement on the wrist. Particularly during jogging or running, a placement of the device on the hip implies more accurate calculation of the total workload.

\section{References}

Anderson T. Biomechanics and running economy. Sports Med, 1996; 22: 76-89

Cohen J. Statistical power analysis for the behavioral sciences. 2nd ed. Hillsdale, NJ: L. Erlbaum Associates; 1988

Coutts AJ, Duffield R. Validity and reliability of GPS devices for measuring movement demands of team sports. J Sci Med Sport, 2010; 13: 133-135

Duthie G, Pyne D, Hooper S. The reliability of video based time motion analysis. J Hum Movement Stud, 2003; 44: $259-271$

Düking P, Hotho A, Fuss FK, Holmberg H, Sperlich B. Comparison of non-invasive individual monitoring of the training and health of athletes with commercially available wearable technologies. Front Physiol, 2016; 7: 71

Field A. Discovering Statistics Using IBM SPSS Statistics (4th ed.). London: Sage Publications Ltd; 2012

Fleming P, Young C, Dixon S, Carré M. Athlete and coach perceptions of technology needs for evaluating 
running performance. Sports Eng, 2010; 13: 1-18

Garmin Ltd. [Internet]. Forerunner 910XT owner's manual. Available from: http://static.garmincdn.com/pumac/Forerunner_910XT_OM_EN.pdf. Accessed on 12.12.2013

Immanuvel Arokia James K, Sudheer K. GSM based multi node pressure sensor in barometric altimeter - a survey. Int J Sci Eng Res, 2011; 2(11)

Menaspa P, Impellizzeri FM, Haakonssen EC, Martin DT, Abbiss CR. Consistency of Commercial Devices for Measuring Elevation Gain. Int J Sports Physiol Perform, 2014; 9: 884-886

Polar Electro Oy [Internet]. Available from: http://www.polar.com/uken/products/maximize_performance/running_multisport/RS800CX. Accessed on 12.12.2013

Roberts S, Trewartha G, Stokes K. A comparison of time-motion analysis methods for field based sports. Int $J$ Sports Physiol Perform, 2006; 1: 388-399

Scarf P. Route choice in mountain navigation, Naismith's rule, and the equivalence of distance and climb. J Sports Sci, 2007; 25: 719-726

Strohrmann C, Harms H, Kappeler-Setz C, Troster G. Monitoring kinematic changes with fatigue in running using body-worn sensors. Inf Technol Biomed, 2012; 16: 983-990

Suunto [Internet]. Available from: http://www.suunto.com/Products/sports-watches/Suunto-Ambit2/SuuntoAmbit2-Black/. Accessed on 12.12.2013

Terrier P, Schutz Y. How useful is satellite positioning system (GPS) to track gait parameters? A review. J Neuroengineering Rehabil, 2005; 2: 28

Thielemans V, Meyns P, Bruijn SM. Is angular momentum in the horizontal plane during gait a controlled variable? Hum Mov Sci, 2014; 34: 205-216

Williams KR, Cavanagh PR. Relationship between distance running mechanics, running economy, and performance. J Appl Physiol, 1987; 63: 1236-1245

\section{Corresponding author:}

\section{Rahel Ammann}

Swiss Federal Institute of Sport Magglingen SFISM, Magglingen, Switzerland

Federal Office of Sport FOSPO

Hauptstrasse 247, CH-2532 Magglingen

Phone: +41 584676321

Fax: +41 584676356

E-mail: Rahel.Ammann@baspo.admin.ch 